\title{
Apoio Matricial como articulador das relaçóes interprofissionais entre serviços especializados e atenção primária à saúde
}

\section{| ${ }^{1}$ Cristiane Pereira de Castro, ${ }^{2}$ Gastão Wagner de Sousa Campos |}

Resumo: Este artigo buscou realizar uma revisão integrativa da produção nacional sobre a metodologia de Apoio Matricial, abrangendo tanto estudos empíricos sobre sua efetividade quanto artigos de revisão teórico-metodológica. Foram utilizados artigos indexados nas bases PubMed, NLM e BVS, no período de 1999 a 2013, em português, inglês e espanhol. Foram incluídos 28 artigos, cuja análise permitiu a identificação de três categorias: 1) Artigos de fundação e consolidação do conceito de Apoio Matricial; 2) Artigos de revisão sobre o Apoio Matricial; 3) Artigos decorrentes de pesquisas empíricas envolvendo o conceito de Apoio Matricial. As publicações evidenciaram que o Apoio Matricial traz uma proposta inovadora e importante para transformação do modelo assistencial. Entretanto, para atingir seus objetivos é necessária a superação de alguns impasses, vários ligados diretamente à implementação de suas diretrizes e outros à superação de problemas estruturais do SUS.

> Palavras-chave: apoio matricial; atenção primária; revisão integrativa.

\author{
1 Universidade Estadual de \\ Campinas (Unicamp). São \\ Paulo-SP, Brasil (crispcastro@ \\ gmail.com) \\ 2 Universidade Estadual \\ de Campinas (Unicamp), \\ São Paulo-SP, Brasil \\ (gastaowagner@mpc.com.br).
}


O Apoio Matricial é uma metodologia para organizar o trabalho interprofissional, tanto em equipes quanto em redes de atenção à saúde (CAMPOS, 1999). Utiliza, além dos conceitos de trabalho compartilhado e de cogestão, o referencial da interdisciplinaridade, visão ampliada do processo saúde-doença-cuidado, em suas dimensões sociais, sanitárias e pedagógicas, e objetiva a construção de corresponsabilidade no cuidado em saúde entre equipes multiprofissionais e profissionais apoiadores especialistas (CAMPOS et al., 2013).

A formulação da metodologia de Apoio Matricial se baseou na tradição teórica dos sistemas de saúde nacionais, públicos e hierarquizados, que propuseram um novo modelo de gestão em saúde, com mecanismos de referência e contrarreferência, interconsultas, protocolos e centros de regulação (ORGANIZAÇÃO PANAMERICANA DE SAÚDE, 1964).

O Apoio Matricial alterou essas metodologias de trabalho, como a interconsulta e os sistemas de referência e contrarreferência, mediante mecanismos de personalização, diálogo, decisão compartilhada, responsabilização e compromisso entre as equipes e os profissionais apoiadores especialistas. Além do cuidado compartilhado, verificou-se que essa metodologia funciona, ao mesmo tempo, como uma forma de educação permamente, uma vez que os profissionais com distintas formaçôes ampliam a comunicação entre eles ao conduzir casos de forma compartilhada.

Essas alterações foram realizadas também levando em consideração reflexões e observações sistemáticas sobre as relações interprofissionais, primeiro, em equipes de saúde mental inseridas na atenção primária e, posteriormente, em outras equipes de Hospitais, Centros de Referência para Aids, reabilitação física da rede pública de Campinas/SP durante a década de noventa (DOMITTI, 2006; CAMPINAS, 2001).

O município de Campinas, desde 1989, criou equipes de saúde mental na atenção básica, objetivando reforçar a sua capacidade de resolver problemas de saúde e ainda construir um novo modelo de cuidado em saúde mental. Posteriormente, a partir da constituição da rede de reabilitação psicossocial, o Apoio Matricial passou também a ser utilizado como metodologia para a relação dos serviços especializados em saúde mental (CAPS) com as unidades de atenção básica (BRAGA-CAMPOS, 2000). Por se tratar de uma metodologia 
para ordenar relações interprofissionais dentro de um mesmo serviço ou na rede de saúde, possível de ser utilizada em várias áreas de saber especializado, depois de um tempo, se expandiu para outras áreas, como a reabilitação física, traumatologia, dermatologia e outros serviços especializados.

Em 2008, após um longo período de debate, o Ministério da Saúde publicou uma nova política, denominada "Núcleos de Apoio à Saúde da Família" (NASF), criada pela Portaria no 154 , de 24 de Janeiro de 2008. Essa política prevê recursos financeiros destinados especificamente para a contratação de profissionais de apoio às equipes de Saúde da Família e que utilizariam metodologia de trabalho baseada no arranjo estrutural Apoio Matricial. Atualmente, o NASF é regulamentado pela Portaria $n^{\circ} 2.488$, de 21 de outubro de 2011, e existem 3.125 NASFs implantados em diversos municípios do país (BRASIL, 2015).

Foi realizada uma revisão bibliográfica relacionando o Apoio Matricial com a produção internacional sobre trabalho interprofissional em saúde sob a denominação "Cuidado Compartilhado e Trabalho Colaborativo" (OLIVEIRA; CAMPOS, 2015).

Nesse estudo, os autores identificaram que a proposta de estabelecer troca de conhecimento e atendimento compartilhado entre os diferentes profissionais das equipes de Atenção Básica e especialistas vem sendo utilizada de forma sistemática no cenário internacional, prioritariamente no tratamento de doenças crônicas, como no tratamento da diabetes e na saúde mental, nos seguintes países: Reino Unido (STARFIELD, 2003), Irlanda (SMITH et al., 2007), Austrália (KELLY et al., 2011), Canadá (VINGILIS et al., 2007) e Espanha (GARCÍA-TALAVERA ESPÍN et al., 2012). Em Portugal, apesar de ainda estar em estágio inicial, foi possível identificar iniciativas de cuidado compartilhado entre profissionais da Psicologia e da Nutrição, que já começaram a desenvolver uma prática pautada pela relação dialógica e colaborativa com as equipes multidisciplinares da Atenção Primária (PISCO, 2011; TRINDADE; TEIXEIRA, 2010).

Dessa forma, partindo dos achados de Oliveira e Campos (2015), este estudo buscou realizar uma revisão integrativa da produção nacional sobre a metodologia de Apoio Matricial, abrangendo tanto estudos empíricos sobre a efetividade quanto artigos de revisão teórico-metodológica sobre o tema.

A revisão que este artigo apresenta compõe uma pesquisa de doutorado do Programa de Pós-Graduação em Saúde Coletiva da Faculdade de Ciências Médicas da UNICAMP. 


\section{Metodologia}

Este estudo foi realizado por meio de uma revisão integrativa (COOPER, 1982), método utilizado para agrupar os resultados de pesquisas primárias sobre o mesmo assunto, com o objetivo de sintetizar e analisar dados para construir uma avaliação sobre o estado da arte da metodologia do Apoio Matricial no Brasil.

A questão norteadora desta revisão foi: quais as contribuiçôes e impasses da aplicação do Apoio Matricial em saúde no Brasil como articulador das relaçóes interprofissionais entre Atenção Primária à Saúde e serviços especializados?

Conforme recomendações, foram definidos os critérios de inclusão e exclusão adotados para a seleção dos estudos. Assim sendo, foram incluídos artigos na íntegra, publicados em português, inglês ou espanhol, que tratassem do tema da pesquisa, contivessem as palavras-chave (atenção primária e Apoio Matricial) e fossem publicados nos últimos 14 anos (1999 a 2013). Os critérios de exclusão foram: artigos incompletos e apenas resumos, trabalhos não disponíveis online e que não traziam abordagem sobre a temática do estudo.

A coleta de dados ocorreu de novembro de 2013 a junho de 2014. Foram acessados artigos publicados desde 1999, ano da formulação da concepção de Apoio Matricial por Gastão Wagner Campos, até 2013, indexados na base PubMed, no serviço da Biblioteca Nacional de Medicina Americana e na Biblioteca Virtual em Saúde, que dá acesso às bases de dados: Literatura LatinoAmericana e do Caribe em Ciências da Saúde, Medical Literature Analysis and Retrieval System Online e Índice Bibliográfico Espanhol em Ciências da Saúde.

Os autores elaboraram um instrumento específico para essa revisão, com o objetivo de organizar e sumarizar as informações de maneira concisa, formando um banco de dados de fácil acesso e manejo. A identificação do estudo, definição dos sujeitos, os objetivos, a metodologia empregada e os principais resultados encontrados são algumas das informações exigidas pelo instrumento.

A análise e a síntese dos dados extraídos dos artigos foram realizadas de forma descritiva, possibilitando observar e classificar os estudos, com o intuito de reunir o conhecimento produzido sobre o tema explorado na revisão.

Esta revisão não apresentou necessidade de aprovação por Comitê de Ética em Pesquisa, uma vez que manipula dados de livre acesso, não se tratando, portanto, de documentos que requeiram sigilo. As demais questôes éticas foram 
preservadas, na medida em que todos os autores consultados foram devidamente referenciados no texto.

\section{Resultados}

Utilizando-se os passos metodológicos descritos, foram identificadas 225 publicações, das quais 49 foram excluídas por repetição; 20 tinham textos incompletos ou não disponíveis; e 128 foram rejeitadas após a primeira análise de leitura do título e resumo por não incluírem atenção primária e Apoio Matricial.

Em síntese, a amostra final desta revisão foi constituída por 28 artigos, sendo que o quadro 1 representa as especificações de cada um dos artigos.

Quadro 1. Relação das publicações que compuseram o estudo, de acordo com código, título, ano de publicação e autores

\begin{tabular}{|c|c|c|c|c|}
\hline Código & Título & Ano & Revista & Autores \\
\hline A1 & $\begin{array}{l}\text { Equipes de referência e apoio } \\
\text { especializado matricial: um ensaio } \\
\text { sobre a reorganização do trabalho } \\
\text { em saúde }\end{array}$ & 1999 & $\begin{array}{l}\text { Ciência \& Saúde } \\
\text { Coletiva }\end{array}$ & Campos \\
\hline A2 & $\begin{array}{l}\text { Apoio Matricial e equipe de } \\
\text { referência: uma metodologia para } \\
\text { gestão do trabalho interdisciplinar } \\
\text { em saúde }\end{array}$ & 2007 & $\begin{array}{l}\text { Cadernos de } \\
\text { Saúde Pública }\end{array}$ & $\begin{array}{l}\text { Campos e } \\
\text { Domitti }\end{array}$ \\
\hline A3 & $\begin{array}{l}\text { Os Caps e o trabalho em rede: } \\
\text { tecendo o Apoio Matricial na } \\
\text { Atenção Básica }\end{array}$ & 2008 & $\begin{array}{l}\text { Psicologia: } \\
\text { Ciência e } \\
\text { Profissão }\end{array}$ & $\begin{array}{l}\text { Bezerra e } \\
\text { Dimenstein }\end{array}$ \\
\hline A4 & $\begin{array}{l}\text { Saúde Mental e atenção Básica } \\
\text { à Saúde: o Apoio Matricial } \\
\text { na construção de uma rede } \\
\text { multicêntrica }\end{array}$ & 2008 & Saúde em Debate & $\begin{array}{l}\text { Figueiredo } \\
\text { e Onocko- } \\
\text { Campos }\end{array}$ \\
\hline A5 & $\begin{array}{l}\text { Implantação do Matriciamento nos } \\
\text { Serviços de Saúde de Capivari }\end{array}$ & 2009 & Saúde e Sociedade & Arona \\
\hline A6 & $\begin{array}{l}\text { O Apoio Matricial em Unidades de } \\
\text { Saúde da Família: experimentando } \\
\text { inovações em saúde mental }\end{array}$ & 2009 & Saúde e Sociedade & $\begin{array}{l}\text { Dimenstein } \\
\text { et al. }\end{array}$ \\
\hline
\end{tabular}

continua... 


\begin{tabular}{|c|c|c|c|c|}
\hline Código & Título & Ano & Revista & Autores \\
\hline A7 & $\begin{array}{l}\text { Saúde Mental na atenção básica à } \\
\text { saúde de Campinas, SP: uma rede ou } \\
\text { um emaranhado? }\end{array}$ & 2009 & $\begin{array}{l}\text { Ciência \& Saúde } \\
\text { Coletiva }\end{array}$ & $\begin{array}{l}\text { Figueiredo } \\
\text { e Onocko- } \\
\text { Campos }\end{array}$ \\
\hline A8 & $\begin{array}{l}\text { O Apoio Matricial na Perspectiva de } \\
\text { Coordenadoras de Equipes de Saúde } \\
\text { da Família }\end{array}$ & 2009 & $\begin{array}{l}\text { Pesquisas } \\
\text { e Práticas } \\
\text { Psicossociais }\end{array}$ & $\begin{array}{l}\text { Dimenstein, } \\
\text { Galvão e } \\
\text { Severo }\end{array}$ \\
\hline A9 & $\begin{array}{l}\text { O olhar das equipes de referência } \\
\text { sobre o trabalho realizado pelo } \\
\text { Apoio Matricial }\end{array}$ & 2011 & $\begin{array}{l}\text { Revista de } \\
\text { Enfermagem da } \\
\text { UFSM }\end{array}$ & Rosa et al. \\
\hline A10 & $\begin{array}{l}\text { Apoio Matricial e Atenção Primária } \\
\text { em Saúde }\end{array}$ & 2011 & Saúde e Sociedade & $\begin{array}{l}\text { Cunha e } \\
\text { Campos }\end{array}$ \\
\hline A11 & $\begin{array}{l}\text { Estratégia de saúde da família, } \\
\text { Núcleo de Apoio à Saúde da } \\
\text { Família e terapia ocupacional: } \\
\text { problematizando as interfaces }\end{array}$ & 2011 & $\begin{array}{l}\text { Revista de Terapia } \\
\text { Ocupacional da } \\
\text { Universidade de } \\
\text { São Paulo }\end{array}$ & $\begin{array}{l}\text { Lancman e } \\
\text { Barros }\end{array}$ \\
\hline A12 & $\begin{array}{l}\text { Apoio Matricial em Saúde do } \\
\text { Trabalhador: tecendo redes na } \\
\text { Atenção Básica do SUS, o caso de } \\
\text { Amparo/ SP }\end{array}$ & 2012 & $\begin{array}{l}\text { Ciência \& Saúde } \\
\text { Coletiva }\end{array}$ & $\begin{array}{l}\text { Santos e } \\
\text { Lacaz }\end{array}$ \\
\hline A13 & $\begin{array}{l}\text { Práticas que integram a saúde mental } \\
\text { à saúde pública: o Apoio Matricial e } \\
\text { a interconsulta }\end{array}$ & 2012 & $\begin{array}{l}\text { Ciência \& Saúde } \\
\text { Coletiva }\end{array}$ & Silveira \\
\hline A14 & $\begin{array}{l}\text { Ferramenta matricial na produção } \\
\text { do cuidado integral na estratégia } \\
\text { saúde da família }\end{array}$ & 2012 & $\begin{array}{l}\text { Acta Paulista de } \\
\text { Enfermagem }\end{array}$ & Jorge et al. \\
\hline A15 & $\begin{array}{l}\text { Cuidado ao paciente com Doença } \\
\text { Renal Crônica no nível primário: } \\
\text { pensando a integralidade e o } \\
\text { matriciamento }\end{array}$ & 2012 & $\begin{array}{l}\text { Ciência \& Saúde } \\
\text { Coletiva }\end{array}$ & Pena et al. \\
\hline A16 & $\begin{array}{l}\text { Avaliação de estratégias inovadoras } \\
\text { na organização da Atenção Primária } \\
\text { à Saúde }\end{array}$ & 2012 & $\begin{array}{l}\text { Revista de Saúde } \\
\text { Pública }\end{array}$ & $\begin{array}{l}\text { Campos et } \\
\text { al. }\end{array}$ \\
\hline
\end{tabular}

continua... 


\begin{tabular}{|c|c|c|c|c|}
\hline Código & Título & Ano & Revista & Autores \\
\hline A17 & $\begin{array}{l}\text { Apoio Matricial como dispositivo } \\
\text { do cuidado em saúde mental na } \\
\text { atenção primária: olhares múltiplos e } \\
\text { dispositivos para resolubilidade }\end{array}$ & 2012 & $\begin{array}{l}\text { Ciência \& Saúde } \\
\text { Coletiva }\end{array}$ & Pinto et al. \\
\hline A18 & $\begin{array}{l}\text { Apoio Matricial em Saúde Mental: } \\
\text { alcances e limites na atenção básica }\end{array}$ & 2012 & Saúde e Sociedade & $\begin{array}{l}\text { Morais e } \\
\text { Tanaka }\end{array}$ \\
\hline A19 & $\begin{array}{l}\text { Metodologia de Apoio Matricial: } \\
\text { interfaces entre a Terapia } \\
\text { Ocupacional e a ferramenta de } \\
\text { organização dos serviços de saúde }\end{array}$ & 2012 & $\begin{array}{l}\text { Cadernos } \\
\text { de Terapia } \\
\text { Ocupacional da } \\
\text { UFSCar }\end{array}$ & $\begin{array}{l}\text { Souza; Ayres } \\
\text { e Marcondes }\end{array}$ \\
\hline A20 & $\begin{array}{l}\text { Acessibilidade e resolubilidade } \\
\text { da assistência em saúde mental: a } \\
\text { experiência do Apoio Matricial }\end{array}$ & 2013 & $\begin{array}{l}\text { Ciência \& Saúde } \\
\text { Coletiva }\end{array}$ & $\begin{array}{l}\text { Quinderé } \\
\text { et al. }\end{array}$ \\
\hline A21 & $\begin{array}{l}\text { O Psicólogo Apoiador Matricial: } \\
\text { Percepçōes e Práticas na Atenção } \\
\text { Básica }\end{array}$ & 2013 & $\begin{array}{l}\text { Psicologia: } \\
\text { Ciência e } \\
\text { Profissão }\end{array}$ & $\begin{array}{l}\text { Freire e } \\
\text { Pichelli }\end{array}$ \\
\hline A22 & $\begin{array}{l}\text { Apoio Matricial em Saúde Mental: } \\
\text { percepção de profissionais no } \\
\text { território }\end{array}$ & 2013 & $\begin{array}{l}\text { Revista de } \\
\text { pesquisa: cuidado } \\
\text { é fundamental }\end{array}$ & $\begin{array}{l}\text { Azevedo; } \\
\text { Gondim e } \\
\text { Silva }\end{array}$ \\
\hline A23 & $\begin{array}{l}\text { Saúde Mental e Atenção Básica à } \\
\text { Saúde: Criação de uma Rede de } \\
\text { Apoio Matricial }\end{array}$ & 2013 & $\begin{array}{l}\text { Unopar Cientifica } \\
\text { Ciências } \\
\text { Biológicas e da } \\
\text { Saúde }\end{array}$ & Bachetti \\
\hline A24 & $\begin{array}{l}\text { Apoio Matricial em saúde mental } \\
\text { na atenção primária à saúde: uma } \\
\text { análise da produção científica e } \\
\text { documental }\end{array}$ & 2013 & $\begin{array}{l}\text { Interface: } \\
\text { Comunicação, } \\
\text { Saúde e Educação }\end{array}$ & Bonfim et al. \\
\hline A25 & $\begin{array}{l}\text { Apoio Matricial em saúde mental: } \\
\text { fortalecendo a saúde da família na } \\
\text { clínica da crise }\end{array}$ & 2013 & $\begin{array}{l}\text { Revista latino- } \\
\text { americana de } \\
\text { psicopatologia } \\
\text { fundamental }\end{array}$ & $\begin{array}{l}\text { Minozzo e } \\
\text { Costa }\end{array}$ \\
\hline A26 & $\begin{array}{l}\text { Apoio Matricial em saúde mental } \\
\text { entre CAPS e Saúde da Família: } \\
\text { trilhando caminhos possíveis }\end{array}$ & 2013 & Psico-USF & $\begin{array}{l}\text { Minozzo e } \\
\text { Costa }\end{array}$ \\
\hline
\end{tabular}

continua... 


\begin{tabular}{|l|l|c|l|l|}
\hline Código & \multicolumn{1}{|c|}{ Título } & Ano & \multicolumn{1}{c|}{ Revista } & \multicolumn{1}{c|}{ Autores } \\
\hline A27 & $\begin{array}{l}\text { Apoio Matricial como ferramenta de } \\
\text { articulação entre a saúde mental e a } \\
\text { atenção primária à saúde }\end{array}$ & 2013 & $\begin{array}{l}\text { Cadernos Saúde } \\
\text { Coletiva }\end{array}$ & $\begin{array}{l}\text { Machado e } \\
\text { Camatta }\end{array}$ \\
\hline A28 & $\begin{array}{l}\text { Núcleo de Apoio à saúde da família: } \\
\text { revisão narrativa sobre o Apoio } \\
\text { Matricial na atenção básica }\end{array}$ & 2013 & $\begin{array}{l}\text { Corpus et } \\
\text { Scientia }\end{array}$ & $\begin{array}{l}\text { Pasquim e } \\
\text { Arruda }\end{array}$ \\
\hline
\end{tabular}

Todos os artigos incluídos nesta revisão estão publicados em português. Com a análise do ano de publicação dos trabalhos incluídos, observamos um grande intervalo de tempo - sete anos - entre a publicação do artigo que define o Apoio Matricial em saúde (CAMPOS, 1999) e os posteriores, conforme identificado no quadro.

Podemos identificar duas hipóteses sobre essa questão. Primeira: isso pode ter se dado pelo fato de que não havia financiamento específico para estimular o Apoio Matricial, limitando sua implantação a municípios onde os gestores fossem sensíveis a esta metodologia (Campinas/ SP, Belo Horizonte/MG, Quixadá/CE, entre outros). A partir de 2003, alguns programas do Ministério da Saúde Humaniza-SUS (BRASIL, 2004a; 2004b) e Saúde Mental (BRASIL, 2003) também incorporaram essa perspectiva. Apesar disso, é somente com a criação do NASF (BRASIL, 2008) que o Ministério da Saúde possibilita o financiamento que estimula a utilização da metodologia de Apoio Matricial na atenção primária à saúde. Segunda hipótese: do ponto de vista da academia e da produção de conhecimento, consideramos que esse tempo prolongado é esperado entre a proposição da metodologia e consequente utilização no cenário do SUS, seguindo-se investigações e, posteriormente, publicações de pesquisas sobre as experimentaçōes da incorporação do Apoio Matricial segundo várias metodologias, predominando estudos qualitativos com base na visão de trabalhadores (ARONA, 2009; ROSA et al., 2011; LANCMAN; BARROS, 2011; PENA et al., 2012; MORAIS; TANAKA, 2012; SOUZA et al., 2012; FREIRE; PICHELLI, 2013; AZEVEDO et al., 2013; MINOZZO; COSTA, 2013a), gestores (BEZERRA; DIMENSTEIN, 2008; DIMENSTEIN et al., 2009a; FIGUEIREDO; ONOCKO-CAMPOS, 2009; SILVEIRA, 2012; BACHETTI, 2013) e usuários e familiares SANTOS; LACAZ, 2012; JORGE et 
al., 2012; ONOCKO-CAMPOS et al., 2012; PINTO et al., 2012; QUINDERÉ et al., 2013) sobre aplicabilidade da metodologia.

Percebemos também, nos artigos analisados, a prevalência de investigações sobre o Apoio Matricial ligadas à sua aplicação metodológica na rede de saúde mental e sua articulação com a Atenção Primária (BEZERRA; DIMENSTEIN, 2008; FIGUEIREDO; ONOCKO CAMPOS, 2009; SILVEIRA, 2012; JORGE et al., 2012; ONOCKO-CAMPOS et al., 2012; PINTO et al., 2012; MORAIS; TANAKA, 2012; QUINDERÉ et al., 2013; FREIRE; PICHELLI, 2013; AZEVEDO et al., 2013; BACHETTI, 2013; MINOZZO; COSTA, 2013a) e uma frequência menor de artigos (07 estudos) que investigam o Apoio Matricial em outros serviços especializados, e ainda nos Núcleos de Apoio a Saúde da Família (ARONA, 2009; DIMENSTEIN et al., 2009b; ROSA et al., 2011; LANCMAN; BARROS, 2011; SANTOS; LACAZ, 2012; PENA et al., 2012; SOUZA et al., 2012).

Outro ponto que vale ressaltar na análise dos artigos é o fato de que a formulação do conceito de apoio não gerou polêmica teórica, conceitual ou metodológica, observando-se apenas 04 artigos sobre o referencial teórico e metodológico do Apoio Matricial (CAMPOS, 1999; CAMPOS; DOMITTI, 2007; FIGUEIREDO; ONOCKO-CAMPOS, 2008; CUNHA; CAMPOS,2011). Percebe-se, nos demais artigos, um esforço para crítica da metodologia com base nos experimentos e investigações, sem, entretanto, haver ruptura com seu referencial epistemológico central.

Destaca-se que a maioria dos artigos que compuseram esta revisão são pesquisas empíricas que investigam o método de Apoio Matricial (BEZERRA; DIMENSTEIN, 2008; ARONA, 2009; DIMENSTEIN et al., 2009a; FIGUEIREDO; ONOCKO-CAMPOS, 2009; DIMENSTEIN et al., 2009b; ROSA et al., 2011; LANCMAN; BARROS, 2011; SANTOS; LACAZ, 2012; SILVEIRA, 2012; JORGE et al., 2012; PENA et al., 2012; ONOCKOCAMPOS et al., 2012; PINTO et al., 2012; MORAIS; TANAKA, 2012; SOUZA et al., 2012; QUINDERÉ et al., 2013; FREIRE; PICHELLI, 2013; AZEVEDO, et al., 2013; BACHETTI, 2013; MINOZZO; COSTA, 2013a; 2013b). Esses estudos utilizaram variadas composiçōes metodológicas, ainda que todos tenham se valido de representação ou narrativas construídas por atores sociais envolvidos com o processo de Apoio Matricial. 
No que se refere aos sujeitos da pesquisa encontramos o descrito no quadro 2:

Quadro 2. Relação das pesquisas empíricas segundo sujeitos investigados na pesquisa e código dos estudos

\begin{tabular}{|l|l|}
\hline \multicolumn{1}{|c|}{ Código } & \multicolumn{1}{c|}{ Sujeitos das pesquisas } \\
\hline A12, A14, A16, & $\begin{array}{l}\text { Profissionais da atenção primária, serviços especializados, } \\
\text { usuários e gestores }\end{array}$ \\
\hline A7, A13, A23 & $\begin{array}{l}\text { Profissionais da atenção primária, serviços especializados e } \\
\text { gestores }\end{array}$ \\
\hline A5, A22, A25, A26 & Profissionais da atenção primária e serviços especializados \\
\hline A3 & Profissionais dos serviços especializados e gestores \\
\hline A6 & Profissionais da atenção primária e gestores \\
\hline A8 & Gestores locais de unidades de atenção primária \\
\hline A11, A19, A21 & Profissionais do NASF \\
\hline A9, A15,A18 & Profissionais da atenção primária \\
\hline
\end{tabular}

Observam-se então, dez estudos que são pesquisas empíricas envolvendo o Apoio Matricial que valorizam a perspectiva dos profissionais envolvidos (ARONA, 2009; ROSA et al., 2011; LANCMAN; BARROS, 2011; PENA et al., 2012; MORAIS;TANAKA, 2012; SOUSA et al.,2012; FREIRE; PICHELLI, 2013; AZEVEDO et al., 2013; MINOZZO; COSTA, 2013a; 2013b) e 06 estudos que incluem a visão dos gestores junto com os profissionais (BEZERRA; DIMENSTEIN, 2008; DIMENSTEIN et al., 2009a; FIGUEIREDO; ONOCKO-CAMPOS, 2009; DIMENSTEIN et al., 2009b; SILVEIRA, 2012; BACHETTI, 2013). Em apenas cinco estudos se percebe a inclusão da perspectiva dos usuários junto com gestores e profissionais (SANTOS; LACAZ, 2012; JORGE et al., 2012; ONOCKO-CAMPOS et al., 2012; PINTO et al., 2012; QUINDERÉ et al., 2013). Essa relativa homogeneidade de estratégias de pesquisa sobre o Apoio Matricial indica a necessidade de se ampliar a gama de estudos para que seja possível avaliar sua eficácia e efetividade. Considere-se também que o impacto desse modelo de relação interprofissional somente poderá ser compreendido valorizando-se os usuários. 
A análise do conjunto de artigos permitiu a construção de três categorias temáticas, percebidas com base no objetivo deste estudo. $\mathrm{O}$ critério de categorização obedeceu à temática principal abordada; logo, as fontes que apresentaram o mesmo contexto ou ideia foram agrupadas segundo as três categorias.

As categoriais levantadas após análise foram organizadas em três temáticas: 1) Artigos de fundação e consolidação do conceito de Apoio Matricial; 2) Artigos de revisão sobre o Apoio Matricial; 3) Artigos decorrentes de pesquisas empíricas envolvendo o conceito de Apoio Matricial. O quadro 3, a seguir, apresenta como foram organizados essa categorização e seus respectivos artigos.

Quadro 3. Relação das categorias temáticas, com seus respectivos estudos codificados e a porcentagem dos estudos presentes em cada categoria

\begin{tabular}{|l|l|l|}
\hline \multicolumn{1}{|c|}{ Categorias } & \multicolumn{1}{c|}{ Código dos artigos } & \multicolumn{1}{c|}{ Porcentagem \% } \\
\hline $\begin{array}{l}\text { Artigos de fundação e } \\
\text { consolidação do conceito de } \\
\text { Apoio Matricial }\end{array}$ & A1, A2, A4, A10 & $14 \%$ \\
\hline $\begin{array}{l}\text { Artigos de revisão sobre o } \\
\text { Apoio Matricial }\end{array}$ & A24, A27, A28 & $11 \%$ \\
\hline $\begin{array}{l}\text { Artigos decorrentes de } \\
\text { pesquisas empíricas } \\
\text { envolvendo o conceito de } \\
\text { Apoio Matricial }\end{array}$ & $\begin{array}{l}\text { A3, A5, A6, A7, A8, A9, A11, } 13, \mathrm{~A} 14, \mathrm{~A} 15, \mathrm{~A} 16, \mathrm{~A} 17, \\
\mathrm{~A} 18, \mathrm{~A} 19, \mathrm{~A} 20, \mathrm{~A} 21, \mathrm{~A} 22, \mathrm{~A} 23,\end{array}$ & $75 \%$ \\
\hline
\end{tabular}

\section{Artigos de fundação e consolidação do conceito de Apoio Matricial}

Em 1999, Campos publicou o artigo que propõe um novo arranjo permanente para os serviços de saúde (atenção básica, especializada, hospital) para reordenação do trabalho em saúde, com base em dois conceitos: equipe de referência e apoio especializado matricial. Ele argumenta sobre a potência desse arranjo para personalizar e desburocratizar as relações interprofissionais. Com essa finalidade, valoriza as diretrizes de vinculo terapêutico, gestão colegiada e transdisciplinaridade das práticas e saberes. 
Nessa proposta, o autor aponta reformas estruturais voltadas para a constituição de equipes e departamentos multiprofissionais, as Unidades de Produção e Equipes de Referência, em substituição à organização tradicional com base em categorias profissionais. É proposta uma rotação dos organogramas em saúde, de modo que os antigos departamentos especializados (verticais) passem a ser horizontais, oferecendo apoio especializado às equipes interdisciplinares (CAMPOS, 1999).

$\mathrm{O}$ mesmo artigo aponta que as equipes de referência devem funcionar com a adscrição de clientela, garantindo-se um sistema de referência e valorizando o vínculo entre profissionais e usuários. A relação terapêutica, horizontal no tempo, passaria então a ser a linha reguladora do processo de trabalho. Sua principal função seria elaborar e aplicar o projeto terapêutico singular, acionando outros profissionais e equipes especializadas, quando necessário.

Já o apoio especializado matricial objetiva alterar a tradicional noção de referência e contrarreferência vigente nos sistemas de saúde, instituindo uma relação interprofissional dialógica e democrática, pautada por desenhos de projetos terapêuticos, em que as responsabilidades da equipe matricial e de referência não são diluídas, mas sim, compartilhadas e claramente definidas (CAMPOS, 1999).

$\mathrm{O}$ autor aponta, ainda, que a instituição desses conceitos e sua incorporação na prática cotidiana não ocorreriam rapidamente, dependendo de modificações organizativas e da criação de processos facilitadores para reconstrução da cultura institucional e para formação de outra subjetividade profissional (CAMPOS, 1999).

No segundo artigo (CAMPOS; DOMITTI, 2007), são retomados os conceitos de equipe de referência e Apoio Matricial, apresentando-os de duas maneiras: eles seriam arranjos organizacionais e também uma metodologia para a gestão do trabalho em saúde, destacando a contribuição desses conceitos para ampliação das possibilidades de realizar-se clínica ampliada e integração dialógica entre distintas especialidades e profissões.

Define-se que o apoiador matricial é um especialista que tem um núcleo de conhecimento e um perfil distinto daquele dos profissionais de referência, mas que pode agregar recursos de saber e mesmo contribuir com intervenções que aumentem a capacidade de resolver problemas de saúde da equipe primariamente 
responsável pelo caso. Dessa forma, o Apoio Matricial busca personalizar os sistemas de referência e contrarreferência, ao estimular e facilitar o contato direto entre referência encarregada do caso e especialista de apoio (CAMPOS; DOMITTI, 2007).

Apresentam-se duas maneiras para o estabelecimento de contato entre referências e apoiadores. O primeiro seria mediante a combinação de encontros periódicos e regulares - a cada semana, quinzena ou mais espaçados - em que se discutiriam casos clínicos selecionados pela equipe de saúde, elaboração de projetos terapêuticos, pactuação de linhas de intervenção e também diálogo sobre temas clínicos, de saúde coletiva ou de gestão do sistema. Já o segundo seria para dar suporte aos casos imprevistos e urgentes que não pudessem esperar a reunião regular, utilizando meios diretos de comunicação, sempre levando em consideração a pactuação de diretrizes de risco e de acesso aos especialistas apoiadores (CAMPOS; DOMITTI, 2007).

O método de Apoio Matricial é composto por dois conceitos operadores (CAMPOS; DOMITTI, 2007). O termo "matricial" indica uma mudança radical de posição do especialista em relação ao profissional que demanda seu apoio. Busca romper com o princípio da hierarquização, em que se prevê uma diferença de autoridade entre quem encaminha o caso e quem o recebe, resultando em uma transferência de responsabilidade e a instituição de uma relação vertical. O emprego do termo "matricial” busca trazer uma lógica mais próxima à ideia de matriz, sugerindo que profissionais de referência e especialistas mantenham uma relação horizontal, e não apenas vertical, como recomenda a tradição dos sistemas de saúde. Já o termo "apoio" sugere uma metodologia para ordenar essa relação entre referência e especialista, não mais com base na autoridade, mas com base em conceitos dialógicos. O termo foi retirado do método Paidéia (CAMPOS, 2000), que cria a figura do apoiador institucional e sugere que, tanto na gestão do trabalho em equipe quanto na clínica, na saúde pública ou nos processos pedagógicos, a relação entre sujeitos com saberes, valores e papeis distintos pode ocorrer de maneira mais dialógica. No caso, o apoiador procura construir de maneira compartilhada com outros interlocutores projetos de intervenção, tanto se valendo de ofertas originárias de seu núcleo de conhecimentos, de sua experiência e visão de mundo, quanto incorporando demandas trazidas pelo outro também em função de seu conhecimento, desejo, interesses e visão de mundo. 
O artigo também apresenta diversos obstáculos e impasses a essa nova metodologia de trabalho: os estruturais, que estão contidos na própria maneira como as organizações de saúde vêm se estruturando e que impedem a implantação de equipe interdisciplinar e sistemas de cogestão; obstáculos decorrentes do excesso de demanda e da carência de recursos, ligados à implantação parcial do SUS; obstáculos políticos e de comunicação, ligados à tradição das organizações de saúde de funcionarem com concentração de poder em certos profissionais e ausência de espaços coletivos para exercitar a gestão colegiada; obstáculos subjetivo e cultural, ligados ao fato de que a subjetividade dominante nas instituições contemporâneas é pautada pela concorrência exacerbada, que induz posturas cristalizadas e um alto grau de desconfiança em relação ao outro; obstáculo ético, se o método de trabalho com base em equipe de referência e Apoio Matricial busca definir de maneira precisa a responsabilidade sanitária, ao mesmo tempo, complica-se o tema da privacidade e do segredo sobre a história do paciente, da família ou de grupos comunitários; e obstáculo epistemológico, que pressupõe a necessidade de algum grau de adesão dos profissionais a incluir um novo paradigma que pense o processo saúde, doença e intervenção de modo mais complexo e dinâmico, não filiado a nenhuma racionalidade exclusiva (CAMPOS; DOMITTI, 2007).

No terceiro artigo (FIGUEIREDO; ONOCKO CAMPOS, 2008), as autoras apontam como essencial a utilização do Apoio Matricial por dois motivos. A integração do CAPS aos serviços da rede básica é um ponto crucial para continuidade da Reforma Psiquiátrica e construção de ações pactuadas entre os serviços com base na territorialidade. Em segundo lugar, apontam que as questões subjetivas transpassam quaisquer problemas de saúde e devem ser abordadas em toda relação terapêutica. Assim, no Apoio Matricial da Saúde Mental, conhecimentos e ações historicamente reconhecidos como inerentes à prática "psi" são ofertados aos profissionais de saúde de uma equipe, de modo a auxiliá-los a ampliar sua clínica e escuta, a acolher o sofrimento psíquico e a lidar com a subjetividade dos usuários. Ou seja, a saúde mental passa a compor a rede matricial de apoio à atenção básica e a outros programas.

A potência do Apoio Matricial em Saúde Mental está em desfazer a delimitação entre as diferentes disciplinas e tecnologias, através das discussões de caso e da regulação defluxo, evitando práticas que levem à "psiquiatrização" eà "medicalização" do sofrimento humano (FIGUEIREDO; ONOCKO-CAMPOS, 2008). 
Por fim, as autoras reconhecem que a mudança da lógica de trabalho proposta pelo Apoio Matricial não é fácil de ser assumida pelas equipes e não ocorre automaticamente. Dessa forma, é de extrema importância a instituição de espaços coletivos destinados à reflexão e à análise crítica sobre o próprio trabalho, que possam ser continentes aos problemas de relação entre a equipe, aos preconceitos em relação à loucura, à dificuldade de entrar em contato com o sofrimento do outro e à sobrecarga trazida pela lida diária com a pobreza e violência (FIGUEIREDO; ONOCKO-CAMPOS, 2008).

O quarto artigo (CUNHA; CAMPOS, 2011), apresenta uma reflexão teórica sobre a utilização do método de Apoio Matricial no NASF (BRASIL, 2008).

Com base nessa metodologia, o Ministério da Saúde previu a inclusão na atenção básica de vários profissionais antes alocados somente em serviços especializados. Suas principais atividades na realização do Apoio Matricial são: atendimento compartilhado, para uma intervenção interdisciplinar, com troca de saberes, capacitação e responsabilidades mútuas, gerando experiência para ambos os profissionais envolvidos; intervenções específicas do profissional NASF com os usuários e/ou famílias, com discussão e negociação a priori com os profissionais da equipe de Saúde da Família responsáveis pelo caso; ações comuns nos territórios de sua responsabilidade desenvolvidas de forma articulada com as equipes de Saúde da Família (BRASIL, 2009).

Os autores advertem que problemas estruturais, como a escassez de serviços especializados, podem induzir à utilização do NASF de forma equivocada, de maneira substitutiva, visto que é comum se observarem situaçóes em que os profissionais NASF são vistos como "tapa buracos" na ausência de um centro de referência especializado. Além disso, na medida em que outros serviços especializados ainda não praticam o Apoio Matricial, o trabalho das equipes NASF ainda é maior, tanto pelo aprendizado mútuo quanto pelo isolamento que a atenção primária experimenta na relação com a rede assistencial (CUNHA; CAMPOS, 2011).

\section{Artigos de revisão sobre o Apoio Matricial}

Através de um estudo de natureza qualitativa do tipo exploratório, incluindo levantamento bibliográfico e uma pesquisa documental, Bonfim et al. (2013) enfatizam as contribuições do Apoio Matricial como eixo articulador entre a saúde 
mental e a atenção primária, embora também indiquem existir impasses entre a lógica hierárquica, burocrática e centrada em procedimentos, predominante nos serviços de saúde e a coexistência com as diretrizes democráticas do Apoio Matricial.

Os autores apontam que o Apoio Matricial surge para confrontar o modelo de atenção burocratizado e centrado na doença. Dessa forma, o Apoio Matricial exige de profissionais e gestores algo além da competência técnica, sendo necessária também a competência relacional, que se configura em uma atitude de abertura para o diálogo e para o saber construído coletivamente, sem, no entanto, descartar o conhecimento nuclear de cada categoria profissional. Processos de cogestão e educação permanente são apontados como facilitadores da implantação adequada do Apoio Matricial (BONFIM et al., 2013).

Por fim, destacam que o Apoio Matricial em saúde mental faz parte de um modelo de atenção à saúde em construção e que novos aportes teórico-práticos e estudos avaliativos são indispensáveis para seu aperfeiçoamento (BONFIM et al., 2013).

Através de uma revisão integrativa, Machado e Camatta (2013) mostram que as publicações evidenciaram contribuiçôes e impasses, pois, embora existam aspectos que dificultam a implantação do Apoio Matricial, este, quando bem utilizado, mostra-se como uma potente ferramenta de interlocução entre a Saúde Mental e atenção primária.

Os principais fatores levantados que dificultam a função do Apoio Matricial como articulador entre saúde mental e atenção primária são: fragilidade da rede de saúde, que compromete o diálogo entre os atores envolvidos no processo de cuidado; dificuldade dos trabalhadores em entender com clareza os princípios e os objetivos do Apoio Matricial; capacitação precária ou inexistente dos profissionais de saúde; desresponsabilização com o usuário, contrária ao que é preconizado pelo Apoio Matricial; e encaminhamento indiscriminado ao especialista sem preocupação com a longitudinalidade do cuidado. Destacamse também problemas relativos à gestão da Saúde Pública, como a escassez de recursos humanos e de serviços, rigidez nas agendas e cobrança por produtividade (MACHADO; CAMATTA, 2013).

Os aspectos positivos na utilização do Apoio Matricial que se apontaram foram: construção coletiva de saberes e melhor comunicação entre trabalhadores, usuários e gestores através da implementação da rede de saúde; uma maior difusão 
do tema da saúde mental através de encontros e trocas cotidianas; aumento da corresponsabilização através do compartilhamento do cuidado, fortalecimento do vínculo entre os profissionais; superação das práticas de medicalização puramente curativas, individuais e hospitalocêntricas; e valorização do agente comunitário de saúde, que passa a ser peça-chave na comunicação entre equipes e usuários (MACHADO; CAMATTA, 2013).

Outro estudo desenvolvido busca identificar os desafios para o Apoio Matricial com o NASF, do ponto de vista dos trabalhadores de saúde, por meio de uma revisão narrativa (PASQUIM;ARRUDA, 2013).

Os resultados dessa revisão mostram apenas impasses. O primeiro é a preocupação dos trabalhadores da saúde com a baixa cobertura, devido à escassez de profissionais, e com a utilização das equipes de Apoio Matricial para cobrir a demanda real de outros níveis de atenção. Em segundo, aponta-se que o NASF enfrenta obstáculos decorrentes de ser um programa recém-criado, mas enfrenta também antigos obstáculos da Saúde Pública, como precarização do trabalho e desrespeito de direitos. Em terceiro lugar, destaca-se que o Apoio Matricial oferecido sem estrutura suficiente, capacidade técnica, recursos humanos e apoio pedagógico, pode se transformar em apenas "mais trabalho", mesmo que as metas sejam atingidas. Por fim, os autores ressaltam que tais impasses são essencialmente de ordem política e que sua superação depende da valorização dos trabalhadores da saúde e da retomada dos princípios da Reforma Sanitária (PASQUIM; ARRUDA, 2013).

\section{Artigos decorrentes de pesquisas empíricas envolvendo o conceito de Apoio Matricial}

Os artigos incluídos nesta categoria apresentam tanto impasses como contribuições do Apoio Matricial como articulador das relações interprofissionais entre atenção primária e serviços especializados.

\section{Principais impasses para implementação do Apoio Matricial}

Conforme já apresentado neste estudo, o conceito de Apoio Matricial surgiu há quinze anos e tem sido incorporado ao SUS desde então. Entretanto, apesar deste fato, observaram-se artigos que apontam essa metodologia para organização do trabalho em saúde como novidade, uma proposta nova, inovadora e com 
implantação variada em território nacional (BEZERRA; DIMENSTEIN, 2008; DIMENSTEIN et al., 2009b) e outros como algo ainda em construção, configurando-se como uma prática anti-hegemônica (BEZERRA; DIMENSTEIN, 2008; LANCMAN; BARROS, 2011).

Sendo o Apoio Matricial uma novidade, identificaram-se resistências dos profissionais, tanto da atenção primária quanto especializada, em incluir essa metodologia no seu cotidiano de trabalho. Para alguns estudos, essa resistência se deve ao fato de que a maioria dos profissionais não conhece o Apoio Matricial e, mesmo quando o conhecem, não o utilizam pela dificuldade em reconhecer sobre suas próprias dificuldades em compreender e aplicar essa metodologia (DIMENSTEIN et al., 2009a; FIGUEIREDO; ONOCKO-CAMPOS, 2009; PINTO et al., 2012; QUINDERÉ et al., 2013). Outros artigos apontam o fato de o Apoio Matricial ser compreendido por alguns profissionais, em especial os da atenção primária, como sobrecarga, mais uma atividade que são obrigados a cumprir. Simplesmente uma transferência de responsabilidades de serviços especializados para atenção básica ou vice-versa, considerou-se que esse discurso está preso à lógica assistencial ambulatorial (a lógica da referência e contrarreferência), e os profissionais não conseguem buscar um novo modo de organização do seu trabalho porque demonstram uma forte adesão ao modelo biomédico (SILVEIRA, 2012; AZEVEDO et al., 2013; MINOZZO; COSTA, 2013a).

Diversos artigos apontam que a lógica de trabalho predominante na maioria dos serviços de saúde é pautada pelo modelo hegemônico tradicional. Assim, a implantação do Apoio Matricial fica difícil porque a gestão municipal organiza o sistema de forma predominantemente hierárquica e favorece a comunicação entre profissionais pautada em protocolos burocráticos, com predomínio do modelo biomédico, medicamentoso e centrado no médico (BEZERRA; DIMENSTEIN, 2008; DIMENSTEIN et al., 2009a; FIGUEIREDO; ONOCKO-CAMPOS, 2009; JORGE et al., 2012; PINTO et al., 2012; MORAIS; TANAKA, 2012; SOUZA et al., 2012; QUINDERÉ et al., 2013)

Outros apontam que a existência de modelos de gestão municipal baseados em produção e metas, particularmente quando a gestão de serviços públicos é realizada por organizações sociais, define um cotidiano de trabalho em que as consultas individuais são priorizadas em detrimento de outras atividades assistenciais, e a presença de diferentes vínculos profissionais cria situações de trabalho heterogêneas, 
trazendo dificuldades para reorganização do trabalho em saúde (LANCMAN; BARROS, 2011; PINTO et al., 2012; MINOZZO; COSTA, 2013b).

É apontado que o Apoio Matricial desenvolvido pelos profissionais não tem seguido necessariamente as definições propostas pelo Ministério da Saúde e está caindo em uma armadilha em que se muda a nomenclatura, mas não o modelo assistencial. Ou seja, estão sendo desenvolvidas ações compatíveis com o modelo hegemônico tradicional (SILVEIRA, 2012; MINOZZO; COSTA, 2013a). Outra armadilha é a implantação parcial do Apoio Matricial, valorizando-se apenas as discussões de casos para realizar encaminhamentos, como se a proposta pudesse ser reduzida apenas a ser a porta de entrada para os atendimentos especializados (FIGUEIREDO; ONOCKO CAMPOS, 2009; ONOCKO-CAMPOS, et al., 2012).

O Apoio Matricial visa ao compartilhamento de saberes através da instituição de uma relação interdisciplinar. Contudo, percebem-se ainda situações em que as ações de matriciamento são centradas em apenas algumas categorias profissionais (médicos, enfermeiros e psicólogos), e isso acaba fortalecendo a prevalência de um único saber em detrimento do leque ampliado de saberes que compõem as equipes de saúde (JORGE et al., 2012; ONOCKO-CAMPOS et al., 2012; SOUZA et al., 2012; QUINDERÉ et al., 2013).

Um impasse na implantação do Apoio Matricial está ligado diretamente à superação dos problemas estruturais e organizacionais que, infelizmente, ainda fazem parte do cotidiano do SUS. Assim, questóes como a sobrecarga de trabalho, dificuldade de compatibilizar as agendas, número de profissionais incompatível com a demanda tanto na atenção primária quanto na especializada, alta rotatividade dos profissionais, infraestrutura inadequada dos serviços, condições de trabalho inadequadas e serviços superlotados (BEZERRA; DIMENSTEIN, 2008; ARONA, 2009; DIMENSTEIN et al., 2009a; FIGUEIREDO; ONOCKOCAMPOS, 2009; LANCMAN; BARROS, 2011; SANTOS; LACAZ, 2012; ONOCKO-CAMPOS et al., 2012), assim como a ausência de uma rede de serviços especializada concreta (compatível com a demanda) e articulada com a rede básica e a inexistência de uma rede de atenção primária eficaz e insuficientes serviços especializados (PENA et al., 2012; PINTO et al., 2012; BACHETTI, 2013), se configuram como questôes-chave que precisam ser superadas para implementação de um modelo de atenção pautado nas necessidades de saúde do usuário e que qualifique a assistência prestada. 
A formação acadêmica das profissões de saúde que valoriza o atendimento isolado de cada profissional e pouco articulado aos princípios do SUS deve necessariamente ser repensada, porque não inclui as diretrizes do Apoio Matricial, é insuficiente para os profissionais atuarem como apoiadores e não ressalta a importância da democratização das relaçōes interprofissionais através da instituição de espaços de cogestão (BEZERRA; DIMENSTEIN, 2008; ARONA, 2009; FIGUEIREDO; ONOCKO-CAMPOS, 2009; ROSA et al., 2011; FREIRE; PICHELLI, 2013; AZEVEDO et al., 2013).

Além de se repensar a formação acadêmica, é também imprescindível conectar a política de formação em serviço com as diretrizes do Apoio Matricial. Alguns estudos apontam que essa prática não vem sendo adotada (DIMENSTEIN et al., 2009a; FIGUEIREDO; ONOCKO-CAMPOS, 2009; AZEVEDO, et al., 2013), dificultando mudanças no processo de trabalho e possibilitando, no cenário das práticas, a coexistência da fragmentação do cuidado e da corresponsabilização.

O apoio da gestão local facilita a implantação do Apoio Matricial. Contudo, até o momento, isso ainda não é uma realidade, e essa ausência de apoio do gestor para incorporação da lógica do matriciamento tem sido mais um impasse. Aponta-se que a implantação dessa metodologia para o trabalho em saúde depende de vontade política. Entretanto, a organização do modelo assistencial muda a cada eleição, e, em algumas situações, o Apoio Matricial é imposto pela gestão, tornando-se cada vez mais nítido o descompasso de tempos da gestão e o ritmo de transformação das praticas cotidianas (BEZERRA; DIMENSTEIN, 2008; FIGUEIREDO; ONOCKO-CAMPOS, 2009; JORGE et al., 2012; AZEVEDO, et al., 2013).

\section{Principais contribuições do Apoio Matricial}

A implantação do Apoio Matricial traz um impacto importante na resolutividade das equipes, ampliando o acesso dos usuários aos serviços de saúde, através da diminuição do número de encaminhamentos desnecessários, e possibilita acordos de classificação de risco, organizando o fluxo de encaminhamentos (ARONA, 2009; FIGUEIREDO; ONOCKO-CAMPOS, 2009; SANTOS; LACAZ, 2012; SILVEIRA, 2012; PENA et al., 2012). 
Outra contribuição dessa metodologia éa sua potência em promover aproximação

entre as equipes através do estreitamento de vínculo entre os profissionais, ampliando as possibilidades de atuação interdisciplinar e de estabelecimento de um novo tipo de relação interprofissional, mais horizontal e dialógica (DIMENSTEIN et al., 2009a; JORGE et al.,2012; QUINDERÉ et al., 2013).

O Apoio Matricial se configura como um elo de interlocução entre profissionais e usuários, proporcionando um maior vínculo e podendo ser também compreendido como uma ferramenta potente para construção da autonomia dos usuários (QUINDERÉ et al., 2013; FREIRE; PICHELLI, 2013). Além disso, o matriciamento também fortalece o acolhimento dos usuários e seus familiares na rede assistencial (SOUZA et al., 2012; MINOZZO; COSTA, 2013a).

A utilização do Apoio Matricial, por meio de suas ferramentas, potencializa a superação do modelo hierarquizado e biomédico, visto que produz uma relação mais democrática e personalizada entre os profissionais (BEZERRA; DIMENSTEIN, 2008; SANTOS; LACAZ, 2012; QUINDERÉ et al., 2013). Instrumentos e ferramentas como a construção compartilhada de Projetos Terapêuticos Singulares (SANTOS; LACAZ, 2012), visitas domiciliares compartilhadas (QUINDERÉ et al., 2013), consultas conjuntas (JORGE et al.,2012) e discussões de casos (MINOZZO; COSTA, 2013a), são apontados como fatores que contribuem para a transformação do modelo assistencial.

Outra contribuição do Apoio Matricial é possibilitar a articulação de uma rede assistencial resolutiva que visa ao cuidado integral através da instituição de corresponsabilidades entre os diversos serviços (BEZERRA; DIMENSTEIN, 2008; DIMENSTEIN et al., 2009a; JORGE et al.,2012; ONOCKO-CAMPOS, et al., 2012) e aproximação dos serviços do território (SILVEIRA, 2012; PINTO et al., 2012).

A reorganização do trabalho em saúde através do Apoio Matricial favorece a implantação da cogestão no cotidiano dos serviços através da instituição de espaços permanentes para repensar o processo de trabalho e possibilitar a transformação das práticas hegemônicas (ARONA, 2009; DIMENSTEIN et al., 2009b; MINOZZO; COSTA, 2013b).

Os profissionais que vivenciam a metodologia do apoio, mesmo enfrentando obstáculos, apresentam postura dinâmica, escapando da paralisia. Dessa forma, 
têm se configurado como importantes atores das transformações necessárias (LANCMAN; BARROS, 2011; SANTOS; LACAZ, 2012; SOUZA et al., 2012).

\section{Considerações finais}

O presente estudo apresenta algumas limitações. Dentre elas, podemos destacar a própria abrangência dos índices de literatura científica. Embora alguns artigos possam não ter sido identificados em nossa revisão, foram utilizados bancos de dados reconhecidos pela comunidade acadêmica.

Os resultados desta revisão mostram que o Apoio Matricial traz uma proposta inovadora e potente para transformação do modelo assistencial, tornandose um importante eixo articulador para produzir mudanças nas relações interprofissionais. Entretanto, para atingir seus objetivos, é necessária a superação de alguns impasses, vários interligados diretamente com a implementação de suas diretrizes e outros com a superação de problemas estruturais do SUS.

Observou-se a necessidade de novos estudos que possibilitem pensar em estratégias de avaliação do Apoio Matricial no SUS para além das representações de gestores e profissionais. São essenciais estudos que considerem resultados e tenham a participação dos usuários e sua opinião acerca do Apoio Matricial.

O Apoio Matricial estimula um novo desenho da rede do SUS, já que, em alguma medida, borra a fronteira entre atenção básica e serviços especializados. Esse projeto fere interesses corporativos e redefine a microfísica do poder nas instituições de saúde. O Apoio Matricial demanda novas estratégias de gestão e novas formas de estruturação das redes em saúde. Essa radicalidade tem despertado adesão entusiástica e resistências entre os trabalhadores de saúde. As dificuldades relatadas são decorrentes menos da efetividade do Apoio Matricial do que da insuficiência de recursos, da predominância da gestão autoritária e do modelo biomédico no SUS. ${ }^{1}$

\section{Referências}

ARONA, E. C. Implantação do matriciamento nos serviços de saúde de Capivari. Saúde e Sociedade. São Paulo, v. 18, supl. 1, p. 26-36, 2009.

AZEVEDO, D. M. et al. Apoio Matricial em saúde mental: percepção de profissionais no território. Rev. Pesq.: cuid. fundam. online. Rio de Janeiro, v. 5, n. 1, p. 3311-3322, 2013. 
BACHETTI, L. S. Saúde mental e atenção básica à saúde: criação de uma rede de Apoio Matricial. UNOPAR Cient. Ciênc. Biol. Saúde. Londrina, v. 15, n. 1, p. 13-19, 2013.

BEZERRA, E.; DIMENSTEIN, M. Os Caps e o trabalho em rede: tecendo o Apoio Matricial na atenção básica. Psicol. Ciênc. e Prof., Brasília, v. 28, n. 3, p. 632-645, 2008.

BONFIM, I. G. et al. Apoio Matricial em saúde mental na atenção primária á saúde: uma análise da produção científica e documental. Interface, Botucatu, v. 17, n. 45, p. 287-300, 2013. BRAGA-CAMPOS, F. C. O modelo da reforma psiquiátrica brasileira e as modelagens de São Paulo, Campinas e Santos. 2000. 177f. Tese (Doutorado em Saúde Coletiva) - Departamento de Medicina Preventiva e Social, Universidade Estadual de Campinas, 2000.

BRASIL. Saúde Mental e Atenção Básica: o vínculo e o diálogo necessários - inclusão das ações de saúde mental na atenção básica, $n^{\circ} 1$. Brasília: Secretaria de Atenção à Saúde; Departamento de Ações Programáticas e Estratégicas; Departamento de Atenção Básica; Coordenação Geral de Saúde Mental e Coordenação de Gestão da Atenção Básica, 2003.

- HumanizaSus: equipe de referência e Apoio Matricial. Brasília: Secretaria Executiva. Núcleo Técnico da Política Nacional de Humanização, $2004 a$.

. HumanizaSus: política nacional de humanização: documento base para gestores e trabalhadores do SUS. Brasília: Secretaria Executiva. Núcleo Técnico da Política Nacional de Humanização, 2004b.

Portaria GM n 154, de 24 de janeiro de 2008. Cria os Núcleos de Apoio à Saúde da Família -NASF. Diário Oficial da União, 04 mar. 2008.

Cadernos de Atenção Básica, no 27 - Diretrizes do NASF -Núcleo de Apoio á Saúde da Família. Brasília: Secretaria de Atenção à Saúde; Departamento de Ações Programáticas e Estratégicas; Departamento de Atenção Básica, 2009.

Portaria no 2.488, de 21 de outubro de 2011. Aprova a Política Nacional de Atenção Básica e prevê alterações na composição da equipe do Núcleo de Apoio a Saúde da Família - NASF. Diário Oficial da União, Brasília, DF, 24 out. 2011.

- Departamento de Atenção Básica. Sala de Apoio à Gestão estratégica. Disponível em: <http://189.28.128.178/sage/>. Acesso em: 04/05/2015.

CAMPINAS. Colegiado de Gestão da Saúde. As Diretrizes da Secretaria Municipal de Saúde - Gestão 2001 - 2004. Projeto Paideia de Saúde da Família. Campinas: Secretaria Municipal de Saúde, 2001.

CAMPOS, G. W. S. Equipes de referência e apoio especializado matricial: um ensaio sobre a reorganização do trabalho em saúde. Ciência e Saúde Coletiva, Rio de Janeiro, v. 4, n. 2, p. 393-403, 1999.

. Um método para análise e cogestão de coletivos. São Paulo: Hucitec, 2000. 
CAMPOS, G. W. S.; DOMITTI, A. C. Apoio Matricial e equipe de referência: uma metodologia para gestão do trabalho interdisciplinar em saúde. Cad. Saúde Pública. Rio de Janeiro, v. 23, n. 2, p. 399-407, 2007.

CAMPOS, G. W. S. et al. Práxis e formação Paideia: apoio e cogestão em saúde. São Paulo: Hucitec, 2013.

COOPER, H. M. Scientific guidelines for conducting integrative research reviews. Rev. Educ. Res., v. 52, n. 2, p. 291-302, 1982.

CUNHA, G. T.; CAMPOS, G. W. S. Apoio Matricial e Atenção Primária em Saúde. Saúde e Sociedade, São Paulo, v. 20, n. 4, p. 961-70, 2011.

DIMENSTEIN, M. et al. O Apoio Matricial em unidade de saúde da família: experimentando inovações em saúde mental. Saúde e Sociedade, São Paulo, v. 18, n. 1, p. 63-74, 2009a.

DIMENSTEIN, M. et al. O apoiador matricial na perspectiva de coordenadoras de equipes de saúde da família. Pesquisa e Práticas Psicossociais, São João Del Rey, v. 4, n. 1, p. 37-48, $2009 \mathrm{~b}$.

DOMITTI, A. C. P. Um possivel diálogo com a teoria a partir das práticas de Apoio Especializado Matricial na Atenção Básica de Saúde. 2006. 87f. Tese (Doutorado em Saúde Coletiva) Departamento de Medicina Preventiva e Social, Universidade Estadual de Campinas, 2006. FIGUEIREDO, M. D.; ONOCKO-CAMPOS, R. Saúde Mental e atenção Básica à Saúde: o Apoio Matricial na construção de uma rede multicêntrica. Saúde em Debate, Rio de Janeiro, v. 32, n. 78-79-80, p. 143-149, 2008.

FIGUEIREDO, M. D.; ONOCKO-CAMPOS, R. Saúde Mental na atenção básica à saúde de Campinas, SP: uma rede ou um emaranhado? Ciênc. Saúde Coletiva, Rio de Janeiro, v. 14, n. 1, p. 129-138, 2009.

FREIRE, F. M. S; PICHELLI, A. A. W. S. O psicólogo apoiador matricial: percepções e práticas na atenção básica. Psicol. Ciênc. e Prof., Brasília, v. 33, n. 1, p. 162-173, 2013.

GARCÍA-TALAVERA ESPÍN, N. V et al. Collaborative Group of the Diabetes Unit of the Reina Sofía University Hospital Murcia, Spain. How to reduce avoidable admissions due to acute diabetes complications? Interrelation between primary and specialized attention in a diabetes unit. Nutr. Hosp., v. 27, n. 6, p. 2079-2088, 2012.

JORGE, M. S. B. et al. Ferramenta matricial na produção do cuidado integral na estratégia saúde da família. Acta Paul. Enferm., São Paulo, v. 25, n. 2, p. 26-32, 2012.

KELLY, B. J. et al. Shared care in mental illness: A rapid review to inform implementation. Int J Ment Health Syst., v. 5, n. 31, 2011.

LANCMAN, S.; BARROS, J. O. Estratégia de saúde da família (ESF), Núcleo de Apoio à Saúde da Família (NASF) e terapia ocupacional: problematizando as interfaces. Rev. Ter. Ocup., Univ. São Paulo, v. 22, n. 3, p. 263-269, 2011 
MACHADO, D. K. S.; CAMATTA, M. W. Apoio Matricial como ferramenta de articulação entre a saúde mental e a atenção primária á saúde. Cad. Saúde Colet., Rio de Janeiro, v. 21, n. 2, p. 224-32, 2013.

MINOZZO, F.; COSTA, I. I. Apoio Matricial em saúde mental: fortalecendo a saúde da família na clínica da crise. Rev. Latinoam. Psicopat. Fund., São Paulo, v. 16, n. 3, p.438450, 2013a.

Apoio Matricial em saúde mental entre CAPS e Saúde da Família: trilando caminhos possíveis. Psico-USF, Bragança Paulista, v. 18, n. 1, p. 151-160, 2013 b.

MORAIS, A. P. P.; TANAKA, O. Apoio Matricial em saúde mental: alcances e limites na atenção básica. Saúde Soc., São Paulo, v. 25, n. 1, p. 48-54, 2012.

OLIVEIRA, M. M.; CAMPOS, G. W. S. Apoios matricial e institucional: analisando suas construções. Ciênc. Saúde Coletiva. Rio de Janeiro, v. 20, n. 1, p. 229-238, 2015.

ONOCKO-CAMPOS, R. et al. Avaliação de estratégias inovadoras na organização da atenção primária á saúde. Rev. Saúde Pública, São Paulo, v. 46, n. 1, p. 43-50, 2012.

ORGANIZAÇÃO PANAMERICANA DE SAÚDE. Informe Dawson sobre el futuro de los servicios médicos y afines - 1920. Publicación Cientifica 93. Washington, DC, 1964.

PASQUIM, H. M.; ARRUDA, M. S. B. Núcleo de apoio à saúde da família: revisão narrativa sobre o Apoio Matricial na atenção básica. Corpus et Scientia, Rio de Janeiro, v. 9, n. 2, p. 34-44, 2013.

PENA, P. F. A et al. Cuidado ao paciente com doença renal crônica no nível primário: pensando a integralidade e o matriciamento. Ciênc. Saúde Coletiva. Rio de Janeiro, v. 17, n. 11, p. 3135-44, 2012.

PINTO A. G. A. et al. Apoio Matricial como dispositivo do cuidado em saúde mental na atenção primária: olhares múltiplos e dispositivos para resolubilidade. Ciênc. Saúde Coletiva. Rio de Janeiro, v. 17, n. 3, p. 653-60, 2012.

PISCO, L. Reforma da Atenção Primária em Portugal em duplo movimento: unidades assistenciais autónomas de saúde familiar e gestão em agrupamentos de Centros de Saúde. Ciên. Saúde Coletiva. Rio de Janeiro, v. 16, n. 6, p. 2841-2852, 2011.

QUINDERÉ, P. H. D e al. Acessibilidade e resolubilidade da assistência em saúde mental: a experiência do Apoio Matricial. Ciênc. Saúde Coletiva. Rio de Janeiro, v. 18, n.7, p. 215766, 2013.

ROSA, F. M. et al. O olhar das equipes de referência sobre o trabalho realizado pelo Apoio Matricial. Rev. Enf. UFSM., Santa Maria, v. 1, n. 3, p. 377-385, 2011.

SANTOS, A. P. L; LACAZ, F. A. C. Apoio Matricial em saúde do trabalhador: tecendo redes na Atenção Básica do SUS, o caso de Amparo/SP. Ciênc. Saúde Coletiva, Rio de Janeiro, v. 17, n. 5, p. 1150-2012, 2012. 
SILVEIRA, E. R. Práticas que integram a saúde mental à saúde pública: o Apoio Matricial e a interconsulta. Ciênc Saúde Coletiva, Rio de Janeiro, v. 17, n. 9, p. 2377-2386, 2012.

SMITH, S. M. et al. Effectiveness of shared care across the interface between primary and specialty care in chronic disease management. Cochrane Database Syst Rev, v. 3, CD004910, 2007.

SOUZA, C. C. B. X. et al. Metodologia de Apoio Matricial: interfaces entre a terapia ocupacional e a ferramenta de organização dos serviços de saúde. Cad. Ter. Ocup., São Carlos, v. 20, n. 3, p. 363-368, 2012.

STARFIELD, B. William Pickles Lecture. Primary and specialty care interfaces: the imperative of disease continuity. Br. J. Gen. Pract., v. 53, n. 494, p. 723-729, 2003.

TRINDADE, I.; TEIXEIRA, J. A. C. Psicologia nos cuidados de saúde primários. Lisboa: Climepsi, 2010.

VINGILIS, E. et al. Descriptive and process evaluation of a shared primary care program. The Internet Journal of Allied Health Sciences and Practice; v. 5, n. 4, 2007.

\section{Nota}

${ }^{1}$ C. P. Castro participou da concepção do artigo, redação, análise e aprovou a versão final a ser publicada. G. W. S. Campos realizou a revisão crítica relevante do conteúdo intelectual e aprovou a versão final a ser publicada. 


\section{Abstract}

\section{Matrix Support as an articulator of interbranch relations between specialized services and primary health care}

This paper sought to carry out an integrative review of the national production on Matrix Support methodology, whilst encompassing empirical studies on effectiveness and theoretical-methodological review papers on Matrix Support. The used material is comprised of papers that are indexed in the PubMed database, the NLM and the BVS (Virtual Library of Health), between 1999 and 2013, in Portuguese, English and Spanish. We included 28 articles, and its analysis allowed the identification of three categories: 1) foundation goods and consolidation of the concept of matrix support; 2) Review articles on the matrix support; 3) Items arising from empirical research involving the concept of matrix support. The publications showed that the matrix support brings an innovative proposal and powerful to transform the health care model. However, to achieve their goals is necessary to overcome some impasses, several interconnected directly with the implementation of their directives and to overcoming structural problems of the National Health System (SUS).

Key words: review literature as topic; matrix support; primary health care. 